\title{
Protrusion on the Depressed Surface of Non-polypoid T1 Colorectal Cancer Is Associated with Venous Invasion
}

\author{
TAKAHIDE SHINAGAWA ${ }^{1}$, KEISUKE HATA ${ }^{1}$, TEPPEI MORIKAWA ${ }^{2}$, HIROTOSHI TAKIYAMA ${ }^{1}$, \\ KENSUKE OTANI $^{1}$, TAKESHI NISHIKAWA ${ }^{1}$, TOSHIAKI TANAKA ${ }^{1}$, TOMOMICHI KIYOMATSU ${ }^{1}$, \\ KAZUSHIGE KAWAI $^{1}$, HIROAKI NOZAWA ${ }^{1}$, SOICHIRO ISHIHARA ${ }^{1}$, \\ HISASHI NAKAMURA ${ }^{3}$, MASASHI FUKAYAMA ${ }^{2}$ and TOSHIAKI WATANABE ${ }^{1}$ \\ Departments of ${ }^{1}$ Surgical Oncology and ${ }^{2}$ Pathology, the University of Tokyo, Tokyo, Japan; \\ ${ }^{3}$ Akasaka Endoscopic Clinic, Tokyo, Japan
}

\begin{abstract}
Aim: The treatment strategies for T1 colorectal cancer $(C R C)$ include both surgical and endoscopic resection. Surgical resection is indicated if lymphovascular invasion is present; however, the endoscopic prediction of lymphovascular invasion has not been reported. We aimed to correlate endoscopic morphology with pathological findings, including lymphovascular invasion, in non-polypoid TI CRC. Materials and Methods: We retrospectively investigated 63 patients with non-polypoid T1 CRC surgically resected between 2008 and 2016. Four typical endoscopic findings related to deep submucosal invasion, namely protrusion from a depressed surface, fold convergence, fullness and hardness, were assessed to elucidate their association with pathological findings. Results: Protrusion was the only finding significantly correlated with positive venous invasion $(67.9 \%$ of the lesions with protrusion vs. $34.3 \%$ of those without protrusion, $p=0.01$ ), which was also confirmed by a multivariable analysis (odds ratio $(O R)=3.72$, 95\% confidence interval $(C I)=1.24-11.2, p=0.02)$. Conclusion: The endoscopic finding of protrusion on a depressed surface may be a sign indicating venous invasion in non-polypoid TI CRC.
\end{abstract}

Among cases of $\mathrm{T} 1$ colorectal cancer (CRC), it has become important to determine the treatment strategy (surgical or endoscopic) accurately based on the endoscopic findings due to advances in techniques for [endoscopic treatment [endoscopic mucosal resection (EMR) or endoscopic submucosal dissection (ESD)] and improvement inendoscopic image quality. The surgical indications for $\mathrm{T} 1$

Correspondence to: Takahide Shinagawa, Department of Surgical Oncology, the University of Tokyo, Address: 7-3-1, Hongo, Bunkyo-ku, Tokyo 113-8655, Japan. Tel: +81 338155411, Fax: +81 338116822, e-mail: takahide124@gmail.com

Key Words: Colorectal cancer, protrusion, depressed surface, venous invasion.
CRC include massive submucosal invasion $(>1,000 \mu \mathrm{m})$, lymphovascular invasion and poorly differentiated adenocarcinoma, signet ring cell carcinoma, or mucinous adenocarcinoma histological type since such factors are strongly associated with lymph node metastasis (1).

Massive submucosal invasion of more than $1,000 \mu \mathrm{m}$ is a well-known risk factor for lymph node metastasis (2); therefore, surgical resection with lymph node dissection should be considered for the curative treatment of lesions with findings of deep submucosal invasion according to the guidelines of the Japanese Society for Cancer of the Colon and Rectum $(3,4)$. In order to determine the depth of invasion, an endoscopic assessment to investigate the tumor morphology is very important. A pit-pattern classification with magnifying colonoscopy is considered to be one of the most effective assessments for predicting invasion depth $(5,6)$. In addition, typical macroscopic findings of CRC have already been reported to be associated with submucosal invasion $(7,8)$.

However, lymphovascular invasion and the histological type of CRC are diagnosed through a pathological examination after endoscopic or surgical resection of lesions and no study has yet successfully predicted these results based on the pre-treatment endoscopic findings. If the pathological risk factors indicative of surgical resection could be detected endoscopically, surgery with lymph node dissection could be more accurately selected as an appropriate treatment strategy for high-risk T1 CRC (9).

Non-polypoid CRC has been reported to carry a higher risk of submucosal invasion than polypoid type, even if the lesions are small in size $(10,11)$. For this reason, further pretreatment assessment of the pathological risk factors for nonpolypoid $\mathrm{T} 1 \mathrm{CRC}$ is expected to influence decisions regarding treatment strategy.

In the present report, to elucidate the endoscopic risk factors indicated for surgical resection in non-polypoid T1 CRC, we retrospectively investigated the relationship between endoscopic and pathological findings of such tumors. 


\section{Materials and Methods}

Patients and endoscopic examinations. A total of 204 patients with early-stage CRC were surgically treated at the Department of Colorectal Surgery, the University of Tokyo Hospital, between January 2008 and December 2016. Among them, we included 87 cases of non-polypoid CRCs in the present study, with polypoid CRCs $(n=107)$ being excluded. The morphology and invasion patterns of laterally spreading tumors (LSTs) are different from, and should be treated separately from, typical non-polypoid CRCs (12). Thus, we excluded 10 cases with LST. We ultimately examined 63 cases in which the depth of invasion was the submucosal layer (T1) and the macroscopic findings were appropriately evaluable. Nonpolypoid CRCs were categorized as type 0-IIa, 0-IIb, 0-IIc, 0IIa+IIc, or 0-IIc+IIa according to the Paris classification of superficial neoplastic lesions (13). Type 0-IIa lesions are only slightly elevated (lower than type 0-I lesions) and are called superficially or slightly elevated. Type 0 -IIb lesions are defined as being completely flat without elevated or depressed areas. Type 0IIc lesions exhibit a shallow absolute depression and are called superficially or slightly depressed. The combined type 0 -IIa+IIc lesions have a superficially elevated protrusion on a shallow depressed surface. Type 0 -IIc+IIa lesions have a shallow depression surrounded by a slightly elevated margin that histologically consists of reactive hyperplastic glands or relatively small protrusions in a depressed area. In both combined type lesions (0-IIa+IIc or 0 IIc+IIa), the elevated components surrounded by a depressed area can be called protrusions on a depressed surface (Figure 1) (14).

In order to elucidate the possible association between the pathological findings of T1 CRC and various endoscopic findings, we selected four endoscopic findings reportedly associated with deep submucosal invasion: protrusions on a depressed surface: one or more irregular protrusions on the surface of a depressed area; fold convergence: two or more mucosal folds converging toward the tumor; fullness: bursting appearance due to expansive growth of the tumor and hardness: the absence of any change in a lesion's morphology $(8,10,15)$ (Figure 2).

All endoscopic findings of tumors were determined by two doctors (one was a Board-certified endoscopist in the Japan Gastroenterological Endoscopy Society, and the other was a nonBoard-certified endoscopist who had performed over 1000 colonoscopies) independently and retrospectively reviewing the preoperative colonoscopy findings with at least four endoscopic images. Chromoendoscopy (CE) with indigo-carmine or crystal violet was additionally used with white-light endoscopy to determine the accurate endoscopic findings for 53 out of the 63 cases. Among 53 cases involving patients who underwent CE, 28 cases were further examined by magnifying colonoscopy and crystal violet staining to assess the invasion depth according to Kudo et al.'s pit-pattern classification (5). The concordance rate of each endoscopic finding between the two doctors was investigated. In cases of disagreement, the final diagnosis was made on discussion between the two doctors. Positive findings were determined based on matching conclusion by the two doctors. All cases were surgically treated with lymph node dissection primarily $(n=55)$ or additionally after EMR or ESD $(n=8)$. This study was approved by the Ethics Committee of the University of Tokyo (no. 3252-5).

Stereomicroscopy. The most recent eight out of 63 cases were further examined using a stereomicroscope (Leica Z6 APO; Leica

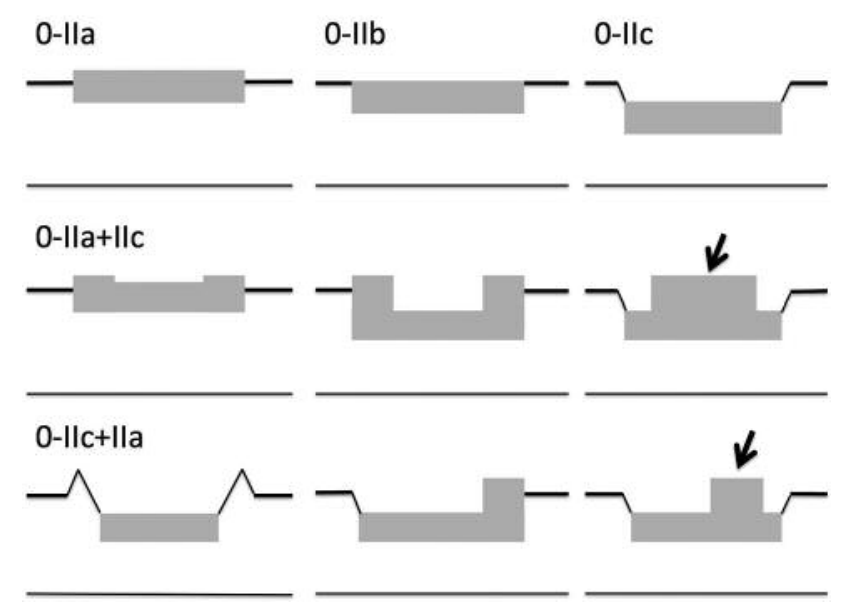

Figure 1. Macroscopic classification of non-polypoid type colorectal cancer. Top row: Type 0-IIa exhibits superficially or slightly elevated lesions. Type 0-IIb has completely flat lesions without elevated or depressed areas. Type 0-IIc exhibits a shallow absolute depression (superficially or slightly depressed). Middle row: Type 0-IIa+IIc lesions have a superficially elevated protrusion on a shallow depressed surface. Bottom row: Type 0-IIc+IIa lesions have a shallow depression surrounded by a slightly elevated margin that histologically consists of reactive hyperplastic glands or relatively small elevated protrusions on a depressed surface. The black arrows indicate the elevated components surrounded by depressed area defined as protrusions on a depressed surface.

Microsystems, Tokyo, Japan). Briefly, the surgical specimens were fixed in $20 \%$ formalin solution, and closely examined under a stereomicroscope. The images of specimens were recorded digitally using a camera mounted on the stereomicroscope (Leica MC170 HD; Leica Microsystems). The lesion in each specimen was also stained with undiluted Carrazzi's hematoxylin solution (Muto Pure Chemicals Co., Ltd., Tokyo, Japan) for 30 seconds in order to make the contrast clear, and all stereomicroscopic findings were assessed using these stained lesions. The lesions were cut into 2-mm-thick slices vertically toward the taenia coli before being embedded in paraffin blocks, and highly experienced pathologists made the histopathological diagnosis. The pathological findings (e.g. histology, invasion depth and lymphovascular invasion) were determined according to the Japanese classification of CRC $(2,3)$. Venous invasion and lymphatic invasion were evaluated with Elastica van Gieson stain and D2-40 stain, respectively.

Statistical analyses. All of the statistical analyses were performed with EZR (16), which is a graphical user interface for $\mathrm{R}$ (https://www.r-project.org/). Nominal variables of groups were compared using the chi-squared test or Fisher's exact test, and continuous variables were compared using the $t$-test. Each test was two-sided, and $p$-values of less than 0.05 were considered statistically significant. The concordance of endoscopic findings for each case between the two endoscopists were quantified by the kappa statistic. Kappa values of more than 0.6 were considered to be substantial, and those more than 0.8 were considered to be almost perfect (17). A multivariable analysis was performed using a logistic regression model. 
Table I. Characteristics of the 63 patients and 63 lesions of nonpolypoid type $T 1$ colorectal cancer.

\begin{tabular}{lc}
\hline Characteristic & \\
\hline Gender, n $(\%)$ & $43(68.3 \%)$ \\
Male & $20(31.7 \%)$ \\
Female & $67.9 \pm 9.12$ \\
Mean age \pm SD, years & $15.8 \pm 5.10$ \\
Mean tumor diameter \pm SD, mm & $2178 \pm 1227$ \\
Mean SM invasion \pm SD, $\mu \mathrm{m}$ & \\
Lymphovascular invasion & $31(49.0 \%)$ \\
Venous & $9(14.3 \%)$ \\
Lymphatic & \\
LN metastasis, n (\%) & $10(15.9 \%)$ \\
Positive & \\
Location, n (\%) & $29(46.0 \%)$ \\
Right & $34(54.0 \%)$ \\
Left & \\
Histology, n $(\%)$ & $54(85.7 \%)$ \\
Well/mod & $6(9.5 \%)$ \\
Poor/sig/muc & $3(4.8 \%)$ \\
Other & \\
Type, n $(\%)$ & $7(11.1 \%)$ \\
0-IIa & $5(7.9 \%)$ \\
0-IIc & $51(81.0 \%)$ \\
0-IIa+IIc/0-IIc+IIa &
\end{tabular}

SM, Submucosal; LN, lymph nodes; Right: cecum, ascending colon, transverse colon; Left: descending colon, sigmoidal colon, rectum; well, well-differentiated adenocarcinoma; mod, moderately differentiated adenocarcinoma; poor, poorly differentiated adenocarcinoma; sig, signet-ring cell carcinoma; muc, mucinous adenocarcinoma; Other: includes papillary adenocarcinoma and medullary adenocarcinoma.

\section{Results}

Clinicopathological characteristics. The clinicopathological characteristics of the 63 cases of non-polypoid T1 CRC examined in this study are shown in Table I. Male patients accounted for $68.3 \%$ of all cases, and the mean age was 67.9 years. The mean tumor diameter was $15.8 \mathrm{~mm}$. The invasion depth of all lesions was the submucosal layer, and most of the tumors had invaded massively into the submucosal layer $(2,178 \pm 1,227 \mu \mathrm{m})$. With regard to lymphovascular invasion, the rates of venous and lymphatic invasion were $49.0 \%$ and $14.3 \%$, respectively. Lymph node metastasis was found in $15.9 \%$ of all cases. The lesions were located slightly more frequently in the left-side colon (descending to sigmoidal colon and rectum; 54.0\%) than the right-sided colon (cecum to transverse colon; $46.0 \%$ ). Most of the tumors were composed of well- to moderately differentiated adenocarcinoma (85.7\%) and macroscopically classified as combined type 0 -IIa+IIc or 0-IIc+IIa lesions (81.0\%).

Endoscopic and pathological findings. Table II shows the association between the four endoscopic findings (protrusion, fold convergence, fullness and hardness) and five pathological findings (submucosal invasion depth, venous and lymphatic invasion, histology, and lymph node metastasis). All of the findings showed substantial to almost perfect kappa values for concordance (protrusion: 28 cases, kappa $=0.72$; fold convergence: 28 cases, kappa $=0.84$; fullness: 15 cases, kappa $=0.88$; hardness: 16 cases, kappa $=0.81$ ). Protrusion was the only finding significantly associated with venous invasion (positive in $67.9 \%$ of the lesions with protrusion $v s .34 .3 \%$ of those without protrusion, $p=0.01$ ), and no other endoscopic findings were significantly correlated with venous invasion. Furthermore, tumors with protrusion or fullness invaded significantly deeper than those without such findings (protrusion: 2,525 vs. 1,900 $\mu \mathrm{m}, p=0.04$; fullness: $2813 v s$. $1979 \mu \mathrm{m}, p=0.02)$. None of the endoscopic findings were significantly associated with lymphatic invasion, histological type or lymph node metastasis, although a tendency toward correlation was noted between fold convergence and histological type (poor, mucinous or signet-ring cell: $17.9 \%$ of the lesions with fold convergence vs. $2.9 \%$ of those without fold convergence, $p=0.08$ ).

A multivariable analysis with a logistic regression model also showed that protrusion was the only significant risk factor for venous invasion (odds ratio $=3.72,95 \%$ confidence interval $=1.24-11.2, p=0.02)($ Table III).

No characteristics or demographics other than venous invasion, submucosal invasion depth and tumor type were significantly different between the groups of tumors with and without protrusion (Table IV).

Diagnostic accuracy. In estimating venous invasion in T1 CRC, the endoscopic finding of protrusion had $61.3 \%$ sensitivity (19/31), 71.9\% specificity (23/32) and $67.9 \%$ positive predictive value (19/28); the overall accuracy was $66.7 \%(42 / 63)$.

Stereomicroscopic observation. Using a stereomicroscope, we further examined the protrusions on depressed surfaces detected by pre-operative colonoscopy and precisely assessed the pathological findings based on thin slices $(2 \mathrm{~mm})$ of the lesions in six out of eight cases examined. Among six of these lesions with protrusion, five had venous invasion, while one out of the two cases without protrusion had venous invasion (Table V). Interestingly, in two cases, venous invasion was observed directly beneath the protruding lesion; the invasion had elevated the surface, resulting in the protrusion. This might explain the association between protrusion and venous invasion in certain cases (Figures 3 and 4).

\section{Discussion}

This is the first study to investigate the correlation between the endoscopic findings and pathological risk factors for surgical indication other than depth of invasion for non- 
Table II. Endoscopic and pathological findings of the cases.

\begin{tabular}{|c|c|c|c|c|c|c|c|c|c|c|c|}
\hline \multirow[b]{2}{*}{$\begin{array}{l}\text { Endoscopic } \\
\text { findings }\end{array}$} & \multicolumn{6}{|c|}{ Pathological findings } & \multicolumn{5}{|c|}{ Histology } \\
\hline & $\begin{array}{c}\mathrm{SM} \text { invasion, } \\
\mu \mathrm{m} \\
\text { mean } \pm \mathrm{SD}\end{array}$ & $p$-Value & $\begin{array}{l}\text { Venous } \\
\text { invasion, } \\
\text { n }(\%)\end{array}$ & $p$-Value & $\begin{array}{c}\text { Lymphatic } \\
\text { invasion, } \\
\text { n (\%) }\end{array}$ & $p$-Value & $\begin{array}{l}\text { Well/mod/ } \\
\text { other, } \\
\text { n }(\%)\end{array}$ & $\begin{array}{c}\text { Poor/sig/ } \\
\text { muc, } \\
\text { n }(\%)\end{array}$ & $p$-Value & $\begin{array}{c}\mathrm{N}+, \\
\mathrm{n}(\%)\end{array}$ & $p$-Value \\
\hline \multicolumn{12}{|l|}{ Protrusion } \\
\hline Positive $\mathrm{N}=28$ & $2,525 \pm 1,255$ & 0.04 & $19(67.9 \%)$ & 0.01 & $2(7.1 \%)$ & 0.28 & $26(92.9 \%)$ & $2(7.1 \%)$ & 0.68 & $4(14.3 \%)$ & $>0.99$ \\
\hline Negative $\mathrm{N}=35$ & $1,900 \pm 1,148$ & & $12(34.3 \%)$ & & $7(20 \%)$ & & $31(88.6 \%)$ & $4(11.4 \%)$ & & $6(17.1 \%)$ & \\
\hline \multicolumn{12}{|c|}{ Fold convergence } \\
\hline Positive $\mathrm{N}=28$ & $2,396 \pm 1,428$ & 0.21 & $14(50 \%)$ & $>0.99$ & $4(14.3 \%)$ & $>0.99$ & $23(82.1 \%)$ & $5(17.9 \%)$ & 0.08 & $4(14.3 \%)$ & $>0.99$ \\
\hline Negative $\mathrm{N}=35$ & $2,003 \pm 1,028$ & & $17(48.6 \%)$ & & $5(14.3 \%)$ & & $34(97.1 \%)$ & $1(2.9 \%)$ & & $6(17.1 \%)$ & \\
\hline \multicolumn{12}{|l|}{ Fullness } \\
\hline Positive $N=15$ & $2,813 \pm 996$ & 0.02 & $10(66.7 \%)$ & 0.15 & $2(13.3 \%)$ & $>0.99$ & $13(86.7 \%)$ & $213.3 \%)$ & 0.62 & $4(26.7 \%)$ & 0.23 \\
\hline Negative $\mathrm{N}=48$ & $1,979 \pm 1,234$ & & $21(43.8 \%)$ & & $7(14.6 \%)$ & & $44(91.7 \%)$ & $4(8.3 \%)$ & & $6(12.5 \%)$ & \\
\hline \multicolumn{12}{|l|}{ Hardness } \\
\hline Positive $N=16$ & $2,566 \pm 1,175$ & 0.15 & $9(56.2 \%)$ & 0.57 & $3(18.8 \%)$ & 0.68 & $14(87.5 \%)$ & $2(12.5 \%)$ & 0.64 & $4(25 \%)$ & 0.26 \\
\hline Negative $N=47$ & $2,046 \pm 1,229$ & & $22(46.8 \%)$ & & $6(12.8 \%)$ & & $43(91.5 \%)$ & $4(8.5 \%)$ & & $6(12.8 \%)$ & \\
\hline
\end{tabular}

SM, Submucosal, LN, lymph nodes; Right: cecum, ascending colon, transverse colon; Left: descending colon, sigmoidal colon, rectum; well, welldifferentiated adenocarcinoma; mod, moderately differentiated adenocarcinoma; poor, poorly differentiated adenocarcinoma; sig, signet-ring cell carcinoma; muc, mucinous adenocarcinoma; Other: includes papillary adenocarcinoma and medullary adenocarcinoma.

polypoid T1 CRC. We showed that tumors with a protruding portion on the depressed surface of non-polypoid T1 CRC had a twofold higher rate of venous invasion than those without such findings $(67.9 \%$ vs. $34.3 \%, p=0.011)$. We also performed a sub-analysis in which 0-IIa lesions were excluded; the results were consistent with the original analysis $(67.9 \%$ vs. $32.1 \%, p=0.015)$, since 0 -IIa lesions do not have a depressed area. Furthermore, this finding was confirmed by a multivariable analysis with a logistic regression model, which suggested the association of venous invasion and the endoscopic finding of protrusion. Further observation and the assessment of surgical specimens using a stereomicroscope were effective in confirming the association between endoscopic appearance and pathological findings. We observed moderate to severe venous invasion right beneath the protruding lesions in two out of six cases (Figures 3 and 4), indicating that protruding region had been directly elevated by the massive venous invasion of cancer in some cases; however, the location of venous invasion was not directly related to that of protrusion in the other four cases.

In our study cohort, most of the tumors invaded massively into the submucosal layer $(2178 \pm 1227 \mu \mathrm{m})$ and showed a higher rate of venous invasion $(49.0 \%)$ and lymph node metastasis $(15.9 \%)$ than in a previous report $(22.4 \%$ and $10.1 \%$, respectively) (2). This might be due to selection bias, as all of the patients were surgically resected, so more advanced cases were selected in our series. However, the mean diameter of the tumors was $15.8 \mathrm{~mm}$, which was smaller than that previously reported for overall T1 CRC cases,
Table III. Multivariable analysis of the endoscopic findings for the risk of venous invasion.

\begin{tabular}{|c|c|c|c|c|}
\hline \multirow[b]{2}{*}{$\begin{array}{l}\text { Endoscopic } \\
\text { findings }\end{array}$} & \multirow[b]{2}{*}{$\begin{array}{c}\text { Venous invasion, } \\
\mathrm{n}(\%)\end{array}$} & \multicolumn{3}{|c|}{ Multivariable analysis } \\
\hline & & $\begin{array}{l}\text { Odds } \\
\text { ratio }\end{array}$ & $\begin{array}{c}95 \% \\
\text { CI }\end{array}$ & $p$-Value \\
\hline \multicolumn{5}{|l|}{ Protrusion } \\
\hline Positive & $19 / 28(67.9 \%)$ & 3.72 & $1.24-11.2$ & 0.02 \\
\hline Negative & $12 / 35(34.3 \%)$ & Reference & & \\
\hline \multicolumn{5}{|c|}{ Fold convergence } \\
\hline Positive & $14 / 28(50.0 \%)$ & 1.35 & $0.446-4.11$ & 0.59 \\
\hline Negative & $17 / 35(48.6 \%)$ & Reference & & \\
\hline \multicolumn{5}{|l|}{ Fullness } \\
\hline Positive & $10 / 15(66.7 \%)$ & 2.87 & $0.725-11.4$ & 0.13 \\
\hline Negative & $21 / 48(43.8 \%)$ & Reference & & \\
\hline \multicolumn{5}{|l|}{ Hardness } \\
\hline Positive & $9 / 16(56.2 \%)$ & 1.40 & $0.386-5.08$ & 0.61 \\
\hline Negative & $22 / 47(46.8 \%)$ & Reference & & \\
\hline
\end{tabular}

95\% CI, 95\% Confidence interval.

including polypoid type $(21.3 \mathrm{~mm})$ (2). This might reflect the fact that non-polypoid CRCs tend to be more invasive than polypoid type, even if they are small in size. Regarding the endoscopic appearance, cases with "protrusion" or "fullness" showed significantly deeper submucosal invasion than those without such findings, suggesting a potential association between the thickness of protrusion or the expansive portion of such tumors and invasion depth. 

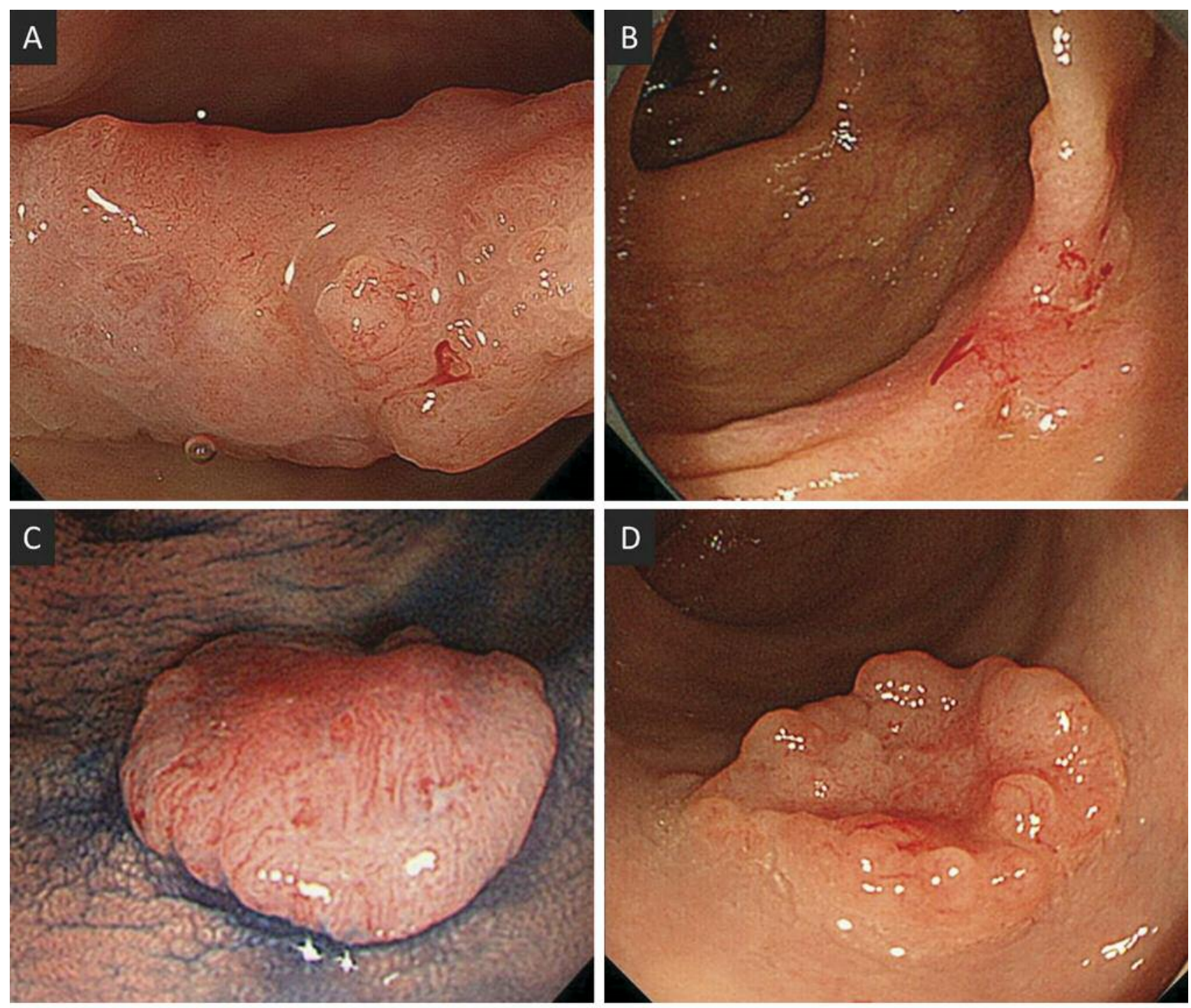

Figure 2. Endoscopic morphology related to deep submucosal invasion. A: Protrusions on a depressed surface: one or more irregular protrusions on the surface of a depressed area. B: Fold convergence: Two or more mucosal folds converging toward the tumor. C: Fullness: A bursting appearance due to expansive growth of the tumor. D: Hardness: The absence of any change in a lesion's morphology.

Regarding the long-term outcomes in our cohort, the recurrence-free survival (RFS) rate was slightly lower in cases with protrusion than in those without, but not to a significant degree (5-year RFS: $100 \%$ without protrusion vs. $92.9 \%$ with protrusion, $p=0.27$ ) (Figure 5). One patient with protrusion developed local recurrence at the anastomotic site without distant metastasis. The long-term outcome of T1 CRC is known to be good, and the relapse rate has been reported to be only $3.7 \%$ (3). However, pre-operative endoscopic resection of high-risk T1 CRC may worsen the outcome, although it is justified for low-risk cases (18). Some studies have suggested that incomplete endoscopic resection of T1 CRC might accelerate the growth of the remaining tumor and promote metastasis (19). Even with complete resection, tumor dissemination was reported to occur in cases of piecemeal resection or perforation $(20,21)$. Therefore, it is vital to detect macroscopic high-risk T1 CRC features, such as protrusion, and avoid inappropriate endoscopic resection of such lesions.

Several limitations associated with the present study warrant mentioning. Firstly, the relatively small number of cases and the likelihood of a certain degree of selection bias should be taken into account because this was a retrospective, singlecenter study. The subjects of the study were all surgically resected cases; among 63 cases, 55 (87.3\%) were primarily 

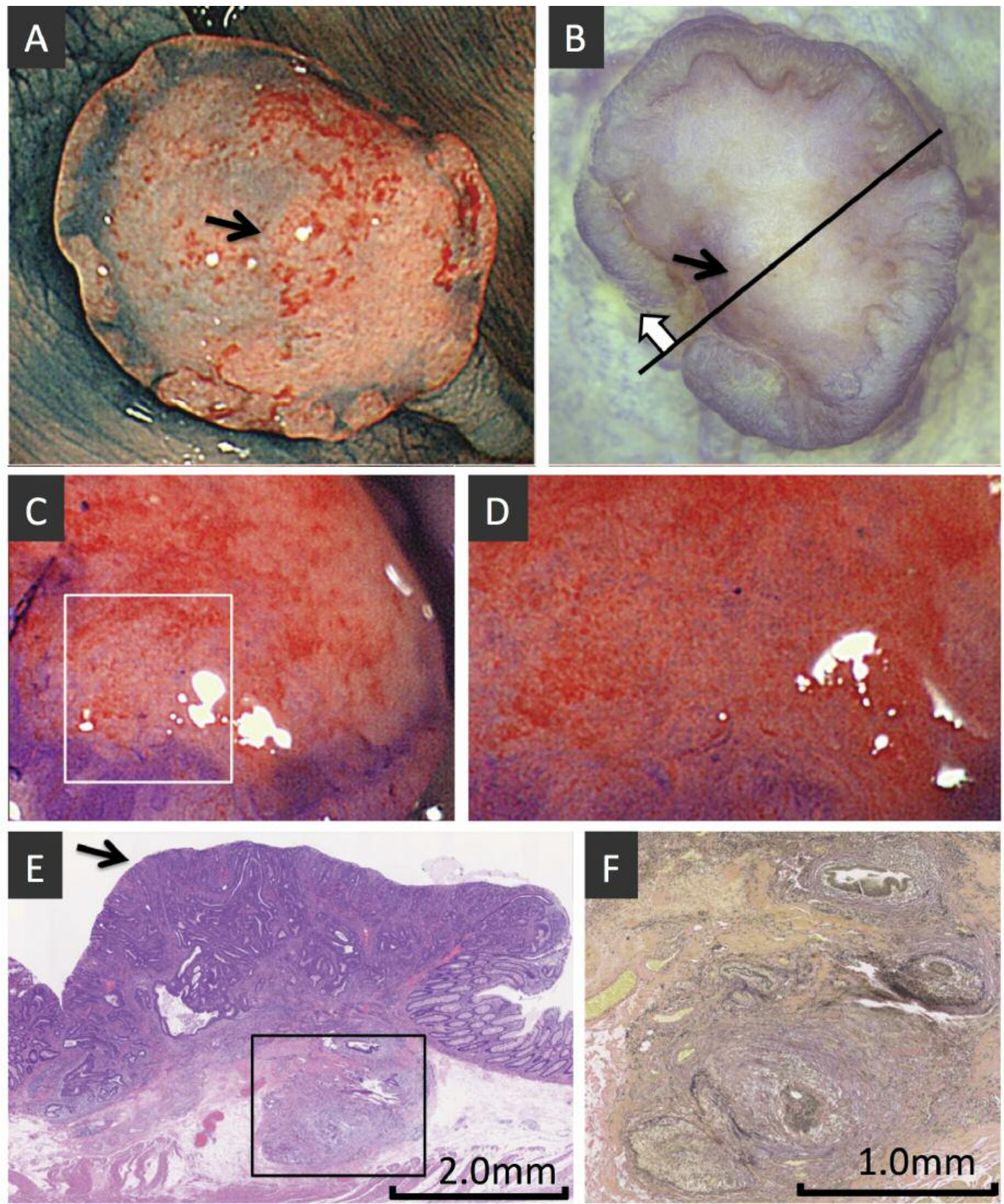

Figure 3. A comparison of the macroscopic and microscopic findings of a case with T1 colorectal cancer (case 1 in Table IV). A: The endoscopic findings with indigo-carmine stain showed that the tumor had a shallow depressed area surrounded by a superficially elevated lesion, which was classified as type 0-IIa+IIc. A protrusion at the center of the depressed surface was noted (arrow). B: In the center of the depressed surface, the same protrusion (black arrow) as the endoscopic finding was detected by stereomicroscopic observation of the tumor in the resected specimen using Carrazzi's hematoxylin staining. C: Crystal violet was used to assess the details of the morphology and pit-pattern of the tumor. D: A magnified view of the region highlighted in C reveals non-structural pits (type VN pattern) in the depressed area, which was considered to be a sign of massive submucosal invasion. E: Microscopic findings of the tumor, which was cut at the black line and observed from the side indicated by the white arrow in $B$, shows that venous invasion at the submucosal layer had elevated the depressed surface of the tumor to form the protrusion (black arrow); hematoxylin-eosin stain. F: Magnified view of the region highlighted in E. The microscopic findings of the tumor with Elastica van Gieson stain showed venous invasion at the submucosal layer. 

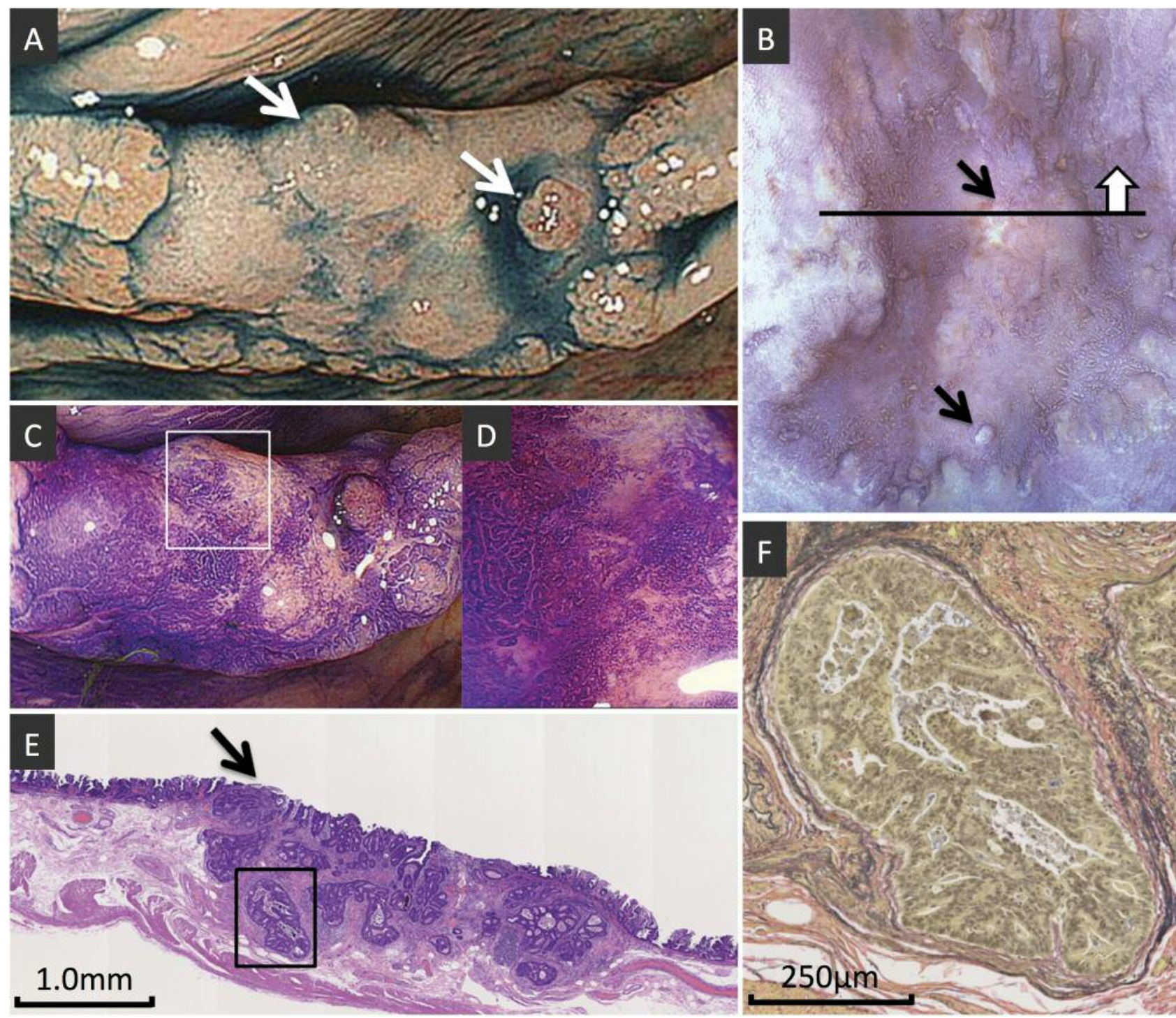

Figure 4. A comparison of the macroscopic and microscopic findings of a case with T1 colorectal cancer (case 3 in Table IV). A: The endoscopic findings with indigo-carmine stain showed that the tumor had absolute depression, which was classified as type 0-IIc. Two slight protrusions on the depressed surface were noted (white arrows). B: The same protrusions as noted on the endoscopic finding were detected by stereomicroscopic observation of the tumor in the resected specimen with Carrazzi's hematoxylin stain (black arrows). C: Crystal violet was used to assess the details of the morphology and pit-pattern of the tumor. D: A magnified view of the region highlighted in C shows the irregular arrangement of pits (type VI pattern with high irregularity) around the protruding portion, which suggests the high possibility of massive submucosal invasion. E: The microscopic findings of the tumor, which was cut at the black line and observed from the side indicated by the white arrow in B, showed that venous invasion at the deep submucosal layer had elevated the depressed surface of the tumor to form the protrusion (black arrow); hematoxylin-eosin stain.F: Magnified view of the region highlighted in E. The microscopic findings of the tumor with Elastica van Gieson stain showed venous invasion at the submucosal layer.

resected, and the other eight $(12.7 \%)$ were additionally resected after endoscopic treatment (EMR or ESD). Therefore, the characteristics of the lesions might have tended to be relatively advanced, and we unable to directly apply the results to the selection of surgical or endoscopic treatment. To evaluate tumor characteristics accurately and select the optimum treatment strategy, further accumulation of cases, including non-surgically treated cases, is warranted. As a further investigation, we assessed 14 tumors that were endoscopically resected during the same period (2008-2015) and found that none of the Tis lesions (10 out of 14 lesions) showed protrusion, fold convergence, fullness or hardness (Table VI). 
Table IV. A comparison of the cases with and without protrusion

\begin{tabular}{lccc}
\hline & \multicolumn{2}{c}{ Protrusion } & \\
\cline { 2 - 3 } Characteristic & With N=28 & Without N=35 & $p$-Value \\
\hline Gender, n (\%) & & & \\
$\quad$ Male & $20(71.4 \%)$ & $23(65.7 \%)$ & 0.79 \\
Female & $8(28.6 \%)$ & $12(34.3 \%)$ & \\
Mean age \pm SD, years & $65.8 \pm 7.01$ & $69.5 \pm 10.3$ & 0.1 \\
Mean tumor diameter \pm SD, mm & $16.2 \pm 3.17$ & $15.4 \pm 6.25$ & 0.55 \\
Mean SM invasion \pm SD, $\mu \mathrm{m}$ & $2525 \pm 1255$ & $1900 \pm 1148$ & 0.04 \\
Lymphovascular invasion & & & \\
Venous & $19(67.9 \%)$ & $12(34.3 \%)$ & 0.01 \\
Lymphatic & $2(7.1 \%)$ & $7(20.0 \%)$ & 0.28 \\
LN metastasis, n $(\%)$ & & & \\
$\quad$ Positive & $4(14.3 \%)$ & $6(17.1 \%)$ & $>0.99$ \\
Location, n (\%) & & & \\
$\quad$ Right & $12(42.9 \%)$ & $17(48.6 \%)$ & 0.8 \\
Left & $16(57.1 \%)$ & $18(51.4 \%)$ & \\
Histology, n (\%) & & & \\
$\quad$ Well/mod & $24(85.7 \%)$ & $30(85.7 \%)$ & 0.64 \\
Poor/sig/muc & $2(7.1 \%)$ & $4(11.4 \%)$ & \\
Other & $2(7.1 \%)$ & $1(2.9 \%)$ & \\
Type, n $(\%)$ & & & \\
0-IIa & $0(0 \%)$ & $7(20.0 \%)$ & 0.03 \\
0-IIc & $2(7.1 \%)$ & $3(8.6 \%)$ & \\
0-IIa+IIc/0-IIc+IIa & $26(92.9 \%)$ & $25(71.4 \%)$ & \\
\hline & & & \\
\hline
\end{tabular}

SM, Submucosal; LN, lymph nodes; Right: cecum, ascending colon, transverse colon; Left: descending colon, sigmoidal colon, rectum; well, well-differentiated adenocarcinoma; mod, moderately differentiated adenocarcinoma; poor, poorly differentiated adenocarcinoma; sig, signet-ring cell carcinoma; muc, mucinous adenocarcinoma; Other: includes papillary adenocarcinoma and medullary adenocarcinoma.

The other four lesions, which invaded the submucosal layer (T1), were completely resected with negative margins (three had no risk factors for lymph node metastasis). In one lesion, submucosal invasion of $2500 \mu \mathrm{m}$ and venous invasion were seen and the patient chose close observation rather than surgical resection. Although one case of fullness and one case of fold convergence were observed in cases involving $\mathrm{T} 1$ tumors, protrusion was only seen in the exceptional case with $2,500 \mu \mathrm{m}$ submucosal invasion and venous invasion. Secondly, the diagnostic accuracy of the endoscopic finding protrusion for venous invasion was not very high $(61.3 \%$ sensitivity, $71.9 \%$ specificity and $67.9 \%$ positive predictive value) even though it was statistically significant as a risk factor for venous invasion. Protrusion alone as a finding should not be used as the diagnostic basis for determining venous invasion, but should instead be used with caution as a finding supportive of such a diagnosis. Finally, in this study we did not investigate all of the cases with protrusion using a stereomicroscope in our evaluation of the correlation of protrusion with venous invasion. Thus, it is necessary to accumulate and evaluate more

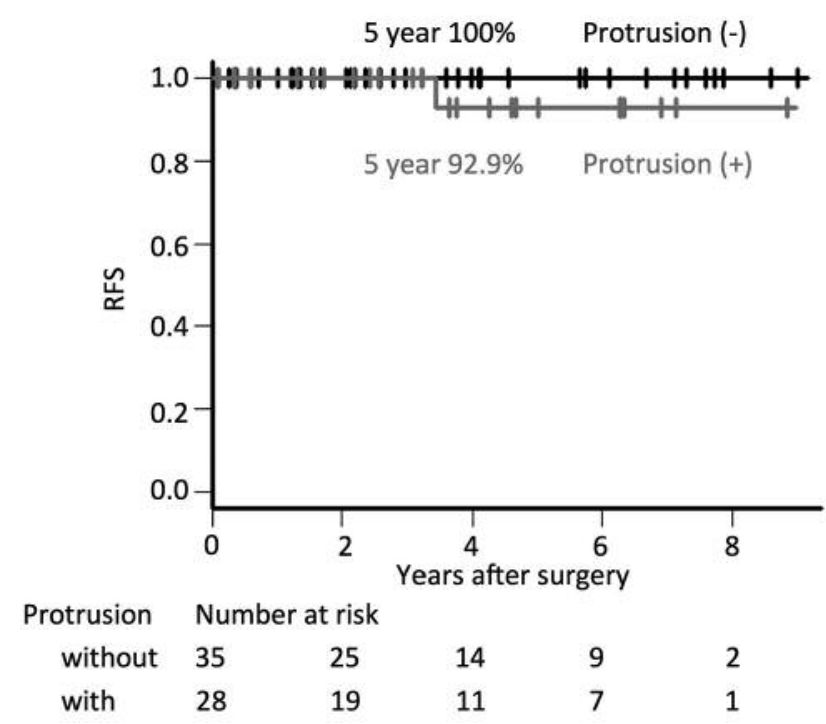

Figure 5. The recurrence-free survival (RFS) of cases with and without protrusion. The RFS rate was slightly lower in the cases with protrusion than in those without (5-year RFS: 100\% without protrusion vs. $92.9 \%$ with protrusion, $p=0.27$ ).

cases prospectively, and to confirm the association between the macroscopic and microscopic findings with close examination of tumor morphology.

Despite these limitations, our study showed that investigating lymphovascular invasion endoscopically in addition to the depth of submucosal invasion might help ensure appropriate decision-making with regard to the treatment strategy for T1 CRC. In our study, it is notable that even in lesions without protrusion, about one-third still harbored venous invasion. Thus, the absence of protrusion alone is not an indication for endoscopic resection. A total assessment, combined with other modalities such as the pit pattern classification or narrow band imaging, is important. We would like to emphasize that white-light observation, including the identification of protrusions, is vital for the assessment of early flat colorectal lesions.

On the other hand, more than $80 \%$ of the lesions with deep submucosal invasion have no lymph node involvement (2); however, no good predictors have been established. At present, the treatment of T1 CRC without lymphovascular invasion is currently a matter of debate. If we are able to improve the prediction of lymph node metastasis, we may be able to avoid unnecessary surgery. Thus, the prediction of venous invasion might help improve decision-making. For this reason, the examination of a protrusion on the depressed surface of a lesion can be considered a supportive endoscopic finding that can be used to detect high-risk T1 $\mathrm{CRC}$ and to assess the need for surgical resection. 
Table V. Characteristics and findings in eight cases examined using stereomicroscopy.

\begin{tabular}{|c|c|c|c|c|c|c|c|c|c|c|c|c|c|}
\hline Case no. & $\begin{array}{c}\text { Age } \\
\text { (years) }\end{array}$ & Gender & Location & Type & Protrusion & $\begin{array}{c}\text { Tumor } \\
\text { diameter } \\
(\mathrm{mm})\end{array}$ & Depth & $(\mu \mathrm{m})$ & Histology & ly & $\mathrm{v}$ & $\mathrm{N}$ & M \\
\hline 1 & 62 & F & A & 0-IIa+IIc & + & 10 & SM & 2,000 & Well & 0 & + & 0 & 0 \\
\hline 2 & 69 & M & $\mathrm{S}$ & 0-IIa+IIc & + & 13 & SM & 1,150 & Mod & 0 & + & 0 & 0 \\
\hline 3 & 74 & $\mathrm{~F}$ & A & 0-IIc & + & 22 & SM & 1,500 & Well & 0 & + & 0 & 0 \\
\hline 4 & 79 & M & $\mathrm{R}$ & 0 -IIa+IIc & + & 19 & SM & 1,500 & Well & 0 & + & 0 & 0 \\
\hline 5 & 75 & F & A & 0-IIa+IIc & + & 14 & SM & 2,800 & Mod & 0 & + & 1 & 0 \\
\hline 6 & 72 & F & A & 0-IIa+IIc & + & 18 & SM & 3,000 & Mod & 0 & 0 & 0 & 0 \\
\hline 7 & 67 & M & $\mathrm{T}$ & 0-IIc & - & 18 & SM & 50 & Well & 0 & 0 & 0 & 0 \\
\hline 8 & 79 & $\mathrm{~F}$ & A & 0 -IIa+IIc & - & 14 & SM & 3,000 & Well & + & + & 1 & 0 \\
\hline
\end{tabular}

A, Ascending colon; T, transverse colon; S, sigmoidal colon; R, rectum; SM, submucosal layer; well, well-differentiated adenocarcinoma; mod, moderately differentiated adenocarcinoma; $\mathrm{v}$, venous invasion; ly, lymphatic invasion; $\mathrm{N}$, lymph node metastasis; $\mathrm{M}$, distant metastasis.

In conclusion, endoscopic findings of a protrusion on the depressed surface of non-polypoid T1 CRC may suggest venous invasion. Therefore, in cases with such findings, surgical resection with lymph node dissection should be considered.

\section{Acknowledgements}

This research was supported by Grants-in-Aid for Scientific Research (A: grant number 16H02672, C: grant number 16K07143, and C: grant number 16K07161) from the Japan Society for the promotion of Science. This research was also supported by the Project for Cancer Research and Therapeutic Evolution (P-CREATE, grant number $16 \mathrm{~cm} 0106502 \mathrm{~h} 0001$ ) from the Japan Agency for Medical Research and Development (AMED).

\section{References}

1 Ueno H, Mochizuki H, Hashiguchi Y, Shimazaki H, Aida S, Hase K, Matsukuma S, Kanai T, Kurihara H, Ozawa K, Yoshimura $\mathrm{K}$ and Bekku S: Risk factors for an adverse outcome in early invasive colorectal carcinoma. Gastroenterology 127 : 385-394, 2004.

2 Kitajima K, Fujimori T, Fujii S, Takeda J, Ohkura Y, Kawamata H, Kumamoto T, Ishiguro S, Kato Y, Shimoda T, Iwashita A, Ajioka Y, Watanabe H, Watanabe T, Muto T and Nagasako K: Correlations between lymph node metastasis and depth of submucosal invasion in submucosal invasive colorectal carcinoma: a Japanese collaborative study. J Gastroenterol 39: 534-543, 2004.

3 Watanabe T, Itabashi M, Shimada Y, Tanaka S, Ito Y, Ajioka Y, Hamaguchi T, Hyodo I, Igarashi M, Ishida H, Ishihara S, Ishiguro M, Kanemitsu Y, Kokudo N, Muro K, Ochiai A, Oguchi M, Ohkura Y, Saito Y, Sakai Y, Ueno H, Yoshino T, Boku N, Fujimori T, Koinuma N, Morita T, Nishimura G, Sakata Y, Takahashi K, Tsuruta O, Yamaguchi T, Yoshida M, Yamaguchi N, Kotake K and Sugihara K: Japanese Society for Cancer of the Colon and Rectum (JSCCR) Guidelines 2014 for treatment of colorectal cancer. Int J Clin Oncol 20: 207-239, 2015.
Table VI. Characteristics of 14 lesions treated with endoscopic mucosal resection.

\begin{tabular}{lc}
\hline Characteristic & \\
\hline Gender, n $(\%)$ & \\
Male & $9(64.3 \%)$ \\
Female & $5(35.7 \%)$ \\
Mean age \pm SD, years & $74.1 \pm 6.20$ \\
Mean tumor diameter \pm SD, mm & $10.1 \pm 4.29$ \\
Invasion depth, n $(\%)$ & \\
M & $10(71.4 \%)$ \\
SM & $4(28.6 \%)$ \\
Mean SM invasion \pm SD, $\mu$ m & $1109 \pm 1012$ \\
Lymphovascular invasion, n $(\%)$ & \\
Venous & $1(7.1 \%)$ \\
Lymphatic & $0(0 \%)$ \\
Location, n $(\%)$ & \\
Right & $4(28.6 \%)$ \\
Left & $10(71.4 \%)$ \\
Histology, n $(\%)$ & \\
Well/mod & $14(100 \%)$ \\
Poor/sig/muc & $0(0 \%)$ \\
Type, n $(\%)$ & \\
$0-I I a$ & $8(57.1 \%)$ \\
$0-I I a+I I c$ & $6(42.9 \%)$ \\
\end{tabular}

M, Mucosal; SM, Submucosal; Right: cecum, ascending colon, transverse colon; Left: descending colon, sigmoidal colon, rectum; well, well-differentiated adenocarcinoma; mod, moderately differentiated adenocarcinoma; poor, poorly differentiated adenocarcinoma; sig, signet-ring cell carcinoma; muc, mucinous adenocarcinoma.

4 Saitoh Y, Inaba Y, Sasaki T, Sugiyama R, Sukegawa R and Fujiya M: Management of colorectal T1 carcinoma treated by endoscopic resection. Dig Endosc 28: 324-329, 2016.

5 Kudo S, Rubio CA, Teixeira CR, Kashida H and Kogure E: Pit pattern in colorectal neoplasia: endoscopic magnifying view. Endoscopy 33: 367-373, 2001. 
6 Tanaka S, Kaltenbach T, Chayama K and Soetikno R: Highmagnification colonoscopy (with videos). Gastrointest Endosc 64: 604-613, 2006.

7 Saitoh Y, Watari J, Fujiya M, Ishikawa C, Inaba Y, Ito T, Moriichi K, Okamoto K, Kogo Y, Goto M and Ohta T: Diagnostic accuracy of the submucosal invasion depth for colorectal submucosal cancers, diagnosis of submucosal invasion depth $1,000 \mu \mathrm{m}$ by conventional colonoscopy. Stomach and Intestine 39: 1350-1356, 2004 (in Japanese with English abstract).

8 Ikehara H, Saito Y, Matsuda T, Uraoka T and Murakami Y: Diagnosis of depth of invasion for early colorectal cancer using magnifying colonoscopy. J Gastroenterol Hepatol 25: 905-912, 2010.

9 Belderbos TD, van Erning FN, de Hingh IH, van Oijen MG, Lemmens VE and Siersema PD: Long-term recurrence-free survival after standard endoscopic resection versus surgical resection of submucosal invasive colorectal cancer: a populationbased study. Clin Gastroenterol Hepatol 15: 403-411, 2017.

10 Saitoh Y, Obara T, Watari J, Nomura M, Taruishi M, Orii Y, Taniguchi M, Ayabe T, Ashida T and Kohgo Y: Invasion depth diagnosis of depressed type early colorectal cancers by combined use of videoendoscopy and chromoendoscopy. Gastrointest Endosc 48: 362-370, 1998.

11 Sakashita M, Aoyama N, Maekawa S, Kuroda K, Shirasaka D, Ichihara T, Kuroda Y, Minami R, Maeda S and Kasuga M: Flatelevated and depressed, subtypes of flat early colorectal cancers, should be distinguished by their pathological features. Int J Colorectal Dis 15: 275-281, 2000.

12 Shigita K, Oka S, Tanaka S, Sumimoto K, Hirano D, Tamaru Y, Ninomiya Y, Asayama N, Hayashi N, Nagata S, Arihiro K and Chayama K: Clinical significance and validity of the subclassification for colorectal laterally spreading tumor granular type. J Gastroenterol Hepatol 31: 973-979, 2016.

13 The Paris endoscopic classification of superficial neoplastic lesions: esophagus, stomach and colon: November 30 to December 1, 2002. Gastrointest Endosc 58: S3-43, 2003.

14 Schlemper RJ, Hirata I and Dixon MF: The macroscopic classification of early neoplasia of the digestive tract. Endoscopy 34: 163-168, 2002.
15 Beppu K, Nagahara A, Terai T, Matsumoto K, Shibuya T, Sakamoto N, Osada T, Kawabe M, Otaka M, Ogihara T and Watanabe S: Clinicopathological characteristics of colorectal cancer less than $10 \mathrm{~mm}$ in diameter and invading submucosa and below. J Gastroenterol Hepatol 25(Suppl 1): S57-61, 2010.

16 Kanda Y: Investigation of the freely available easy-to-use software 'EZR' for medical statistics. Bone Marrow Transplant 48: 452-458, 2013.

17 Landis JR and Koch GG: The measurement of observer agreement for categorical data. Biometrics 33: 159-174, 1977.

18 Overwater A, Kessels K, Elias SG, Backes Y, Spanier BW, Seerden TC, Pullens HJ, de Vos Tot Nederveen Cappel WH, van den Blink A, Offerhaus GJ, van Bergeijk J, Kerkhof M, Geesing JM, Groen JN, van Lelyveld N, Ter Borg F, Wolfhagen F, Siersema PD, Lacle MM and Moons LM: Endoscopic resection of high-risk T1 colorectal carcinoma prior to surgical resection has no adverse effect on long-term outcomes. Gut, 2016. doi: 10.1136/gutjnl-2015-310961. [Epub ahead of print].

19 Kikuchi R, Takano M, Takagi K, Fujimoto N, Nozaki R, Fujiyoshi $\mathrm{T}$ and Uchida $\mathrm{Y}$ : Management of early invasive colorectal cancer. Risk of recurrence and clinical guidelines. Dis Colon Rectum 38: 1286-1295, 1995.

20 Koch M, Kienle P, Sauer P, Willeke F, Buhl K, Benner A, Lehnert T, Herfarth C, von Knebel Doeberitz M and Weitz J: Hematogenous tumor cell dissemination during colonoscopy for colorectal cancer. Surg Endosc 18: 587-591, 2004.

21 Jorgren F, Johansson R, Damber L and Lindmark G: Oncological outcome after incidental perforation in radical rectal cancer surgery. Int J Colorectal Dis 25: 731-740, 2010.

Received November 1, 2017

Revised November 19, 2017

Accepted November 23, 2017 